\title{
Mild hypothermic circulatory arrest with selective cerebral perfusion in open arch surgery
}

\author{
Song-Bo Dong", Kai Zhang", Kai Zhu, Long-Fei Wang, Jun Zheng, Jian-Rong Li, Yong-Min Liu, \\ Li-Zhong Sun, Xu-Dong Pan
}

Department of Cardiovascular Surgery, Beijing Anzhen Hospital, Capital Medical University, and Beijing Institute of Heart, Lung, and Blood Vessel Diseases, Beijing, China

Contributions: (I) Conception and design: SB Dong, XD Pan; (II) Administrative support: LZ Sun, YM Liu; (III) Provision of study materials or patients: XD Pan, LZ Sun; (IV) Collection and assembly of data: SB Dong, K Zhang, K Zhu; (V) Data analysis and interpretation: J Zheng, JR Li; (VI) Manuscript writing: All authors; (VII) Final approval of manuscript: All authors.

\#These authors contributed equally to this work.

Correspondence to: Xu-Dong Pan, MD. Department of Cardiovascular Surgery, Beijing Anzhen Hospital, Capital Medical University, Beijing Institute of Heart, Lung, and Blood Vessel Diseases, Beijing 100029, China. Email: spjrd@aliyun.com.

Background: This study aimed to evaluate whether the use of mild hypothermic circulatory arrest (HCA) with selective cerebral perfusion (SCP) in open arch procedure provides comparable perioperative results to moderate HCA for patients with dissected or degenerative arch pathologies.

Methods: Between January 2017 and September 2020, a total of 88 consecutive patients (mean age $47 \pm 11$ years, 71 males) underwent open arch repair under a single surgeon at our institution with mild or moderate systemic hypothermia assisted by unilateral or bilateral SCP. Patients were divided into groups according to the nasopharyngeal temperature at the beginning of HCA: a moderate HCA group ( $n=47$, $53.4 \%)$ and a mild HCA group $(n=41,46.6 \%)$. The postoperative mortality, morbidity, and visceral organ functions between these groups were analyzed retrospectively.

Results: Compared to the moderate HCA group, the mild HCA group had a significantly higher core temperature (nasopharynx: $24.4 \pm 0.8$ vs. $28.5 \pm 2, \mathrm{P}<0.001$; bladder $25.9 \pm 0.9$ vs. $30 \pm 1.2, \mathrm{P}<0.001$ ), and the incidence of major adverse events (MAE) in this group was markedly lower $(21.3 \%$ vs. 4.9\%, $\mathrm{P}=0.031)$. No differences were identified between the two groups refer to in-hospital mortality, permanent neurological deficit (PND), temporary neurological deficit (TND), and paraplegia (8.5\% vs. $2.4 \%, \mathrm{P}=0.366 ; 8.5 \%$ vs. 0 , $\mathrm{P}=0.120 ; 6.4 \%$ vs. $7.3 \%, \mathrm{P}=1.0 ; 4.3 \%$ vs. $2.4 \%, \mathrm{P}=1.0$, respectively). In the moderate HCA group, 6 patients $(12.8 \%)$ developed acute renal failure needing replacement therapy, which did not occur in the mild HCA group ( $\mathrm{P}=0.028)$. The duration of ventilator support and intensive care unit stay was shorter in the mild HCA group, as well as a decreased volume of drainage during the first $24 \mathrm{~h}$ and reduced platelet transfusion.

Conclusions: The preliminary results of the mild HCA group with SCP applied in open arch repair, mainly in total arch replacement (TAR) and stented elephant trunk (SET) implantation for aortic dissection, were satisfactory. Furthermore, comparable inferior outcomes were obtained with mild HCA compared with that of the conventional moderate HCA strategy. These encouraging surgical and postoperative results favor this more aggressive hypothermia strategy in open arch repair.

Keywords: Mild hypothermic circulatory arrest (mild HCA); moderate hypothermic circulatory arrest (moderate HCA); type A aortic dissection (TAAD); open arch repair

Submitted Nov 24, 2020. Accepted for publication Feb 03, 2021.

doi: $10.21037 /$ jtd-20-3550

View this article at: http://dx.doi.org/10.21037/jtd-20-3550 


\section{Introduction}

Arch surgery is one of the major challenges in modern aortic surgery, especially for complex arch pathologies or acute type A aortic dissection (TAAD). Special cerebral and visceral organ protection is mandatory in open arch repair to improve clinical safety. Deep hypothermic circulatory arrest (HCA), introduced by Griepp et al. in the mid-1970s (1), allowed for a short period of circulatory arrest and was considered an essential component of open arch surgery. The application of selective cerebral perfusion (SCP), which was first introduced by Bachet and Kazui (2-4), is an important adjunct to deep HCA. It provided a near physiological method of cerebral perfusion, allowed for a relative higher core temperature during distal anastomosis, and offered a key advantage in reducing mortality and morbidity in the aortic arch field.

Numerous studies have established the superiority of SCP over deep HCA alone, specifically due to its better survival and neurological results, especially in complex cases that involve time-consuming arch repair (5-7). However, there is currently no consensus regarding the optimal level of hypothermia that cardiopulmonary bypass (CPB) can be discontinued in arch repair. The safe limits of distal HCA at higher temperatures have not yet been clearly defined and are not well recognized in clinical practice.

Herein, we performed a retrospective comparative study to explore the safety and efficacy of mild HCA applied in a cohort consisting mainly of TAAD patients treated with total arch replacement (TAR) and stented elephant trunk (SET) implantation. We present the following article in accordance with the STROBE reporting checklist (available at http://dx.doi.org/10.21037/jtd-20-3550).

\section{Methods}

All procedures performed in this study involving human participants were in accordance with the Declaration of Helsinki (as revised in 2013). This study was approved by the Ethics Committee of Beijing Anzhen Hospital, Capital Medical University. Written informed consent was obtained for each participant.

\section{Patients}

From January 2017 to September 2020, a total of 88 patients underwent open arch repair under a single surgeon (XD Pan) at Beijing Anzhen Hospital. According to the nasopharyngeal temperature at the beginning of HCA, patients were categorized as mild and moderate HCA groups according to the international consensus guidelines (8): (I) the moderate HCA group: $24.4^{\circ} \mathrm{C}(\mathrm{n}=47,53.4 \%)$; (II) the mild HCA group: $28.5^{\circ} \mathrm{C}(\mathrm{n}=41,46.6 \%)$. In almost all cases, the bladder temperature was approximately $1-2{ }^{\circ} \mathrm{C}$ higher than the nasopharyngeal temperature. The mean bladder temperature during open arch repair in the moderate HCA group was $25.9^{\circ} \mathrm{C}$, and was $30^{\circ} \mathrm{C}$ in the mild HCA group. SCP was applied for cerebral protection in all cases.

The relevant clinical characteristics of the patients are summarized in Table 1. The patient cohort consisted of 71 males and 17 females, with a mean age of 47 years (range, 19-71 years). Indications for arch repair were TAAD in 84 patients, type B dissection with root aneurysm in three patients, and degenerative aortic aneurysm in one patient. The most prevalent comorbidity was arterial hypertension, which was diagnosed in 72 patients $(81.8 \%)$, followed by smoking in $47(53.4 \%)$ and pericardial effusion in 42 (47.7\%). Severe or moderate aortic regurgitation was found in 25 patients $(28.4 \%)$, malperfusion in 12 cases (13.6\%), chronic kidney disease in 10 cases (11.4\%), and cardiac tamponade in 8 cases $(9.1 \%)$.

Transthoracic echocardiography and computed tomography angiography (CTA) were routinely conducted preoperatively for confirmation of diagnosis, to identify involved aortic valves and pericardial effusion, and to evaluate cardiac function. Survivors were followed by clinic visits mainly. The referring physician recorded survival, re-intervention, and adverse events. Follow-up echocardiography and CTA were suggested annually.

\section{Definitions}

Death occurring in the hospital during the perioperative period was defined as in-hospital mortality. Permanent neurological deficit (PND) was identified as a newly developing neurological deficit verified by cranial CT scan. Temporary neurological deficit (TND) referred to the occurrence of reversible delirium, agitation, confusion, or motor deficit, which was resolved before discharge (verified by a normal CT scan). The definition of postoperative acute kidney injury (AKI) was cited from the Kidney Disease Improving Global Outcomes criteria (9), and was diagnosed when the value of postoperative creatinine was 1.5 -fold higher than the baseline level or an increase in creatinine of $0.3 \mathrm{mg} / \mathrm{dL}$ occurred within $48 \mathrm{~h}$ postoperatively. Major adverse events (MAE) referred to any of the following 
Table 1 Preoperative clinical profiles

\begin{tabular}{|c|c|c|c|}
\hline Variables & Moderate HCA group $(n=47)$ & Mild HCA group $(n=41)$ & $P$ value \\
\hline Male & $40(85.1 \%)$ & $31(75.6 \%)$ & 0.26 \\
\hline Acute TAAD & $36(76.6 \%)$ & $36(87.8 \%)$ & 0.174 \\
\hline Chronic TAAD & $10(21.3 \%)$ & $2(4.9 \%)$ & 0.031 \\
\hline Type B aortic dissection with root aneurysm & $1(2.1 \%)$ & $2(4.9 \%)$ & 0.596 \\
\hline Hypertension & $39(83 \%)$ & $33(80.5 \%)$ & 0.762 \\
\hline Smoking & $26(55.3 \%)$ & $21(51.2 \%)$ & 0.701 \\
\hline Malperfusion & $6(12.8 \%)$ & $6(14.6 \%)$ & 0.799 \\
\hline Diabetes mellitus & $1(2.1 \%)$ & $4(9.8 \%)$ & 0.18 \\
\hline Marfan syndrome & $2(4.3 \%)$ & $4(9.8 \%)$ & 0.411 \\
\hline Chronic kidney disease & $6(12.8 \%)$ & $4(9.8 \%)$ & 0.745 \\
\hline Dialysis & 0 & $1(2.4 \%)$ & 0.466 \\
\hline Previous cerebrovascular history & $4(8.5 \%)$ & $1(2.4 \%)$ & 0.366 \\
\hline Aortic regurgitation (moderate or severe) & $18(38.3 \%)$ & $7(17.1 \%)$ & 0.028 \\
\hline Ejection fraction & $61.1 \pm 5$ & $62 \pm 5.7$ & 0.462 \\
\hline \multicolumn{4}{|l|}{ Admission laboratory data } \\
\hline
\end{tabular}

Continuous data are presented as mean $\pm \mathrm{SD}$ or median [interquartile range (IQR)], and categorical data are presented as the $\mathrm{n}(\%)$. HCA, hypothermic circulatory arrest; TAAD, type A aortic dissection.

conditions: paraplegia, PND, continuous renal replacement therapy (CRRT) for acute renal failure, and in-hospital mortality.

\section{Surgical techniques}

The hypothermic and SCP strategy has been evolving in recent years. Unilateral selective cerebral perfusion (uSCP) with moderate HCA was the primary method of choice between 2017 and 2019. After 2019, there was a trend toward bilateral selective cerebral perfusion (bSCP) and mild HCA. Sun's procedure has been applied widely in open arch repair for treating extensive arch pathology in indicated patients at our institution, and the technique involving TAR with SET implantation has been reported in our previous study $(10,11)$. Indications for TAR in this study referred to either of the following conditions: atherosclerotic aneurysm in the arch and descending aorta, aortic root aneurysm complicated by type B aortic dissection with involved arch, as well as TAAD with involved arch vessels, a primary arch tear, intimal intussusception in the arch, and Marfan syndrome. In patients with uSCP and moderate HCA, the right axillary artery was cannulated for CPB and SCP. The procedure involved implantation of a SET (Cronus, MicroPort, China) in the descending aorta, followed by TAR with a four-branched graft (Vascutek, Terumo, Japan). After completing the distal arch anastomosis, lower body reperfusion was performed. To minimize the cerebral and 
coronary ischemia, the sequence of aortic reconstruction was special (i.e., proximal descending aorta, then left carotid artery, then ascending aorta, then left subclavian artery, and finally, innominate artery).

In cases with bSCP and mild HCA, the supra-aortic vessels were clamped once the target temperature was reached. Bilateral hemispheres were perfused via the right axillary and left common carotid artery. Next, we implanted the SET and performed distal arch anastomosis. Lower body reperfusion was subsequently resumed. We anastomosed the ascending aorta firstly, and then the left subclavian artery, the left carotid artery, and the innominate artery finally.

\section{Statistical analysis}

All perioperative data were collected prospectively. Statistical analysis was performed using SPSS 22.0 (SPSS, Inc., Chicago, IL, USA). The normality of continuous data was evaluated by Kolmogorov-Smirnov test. Data with normal distribution were expressed as mean \pm standard deviation (SD) and were assessed by the Student's $t$-test. Median and interquartile range were applied to describe non-normally distributed data, and the Mann-Whitney U-test was performed for comparing. Chi-squared or Fisher's exact tests were applied for categorical variables. The mixed effect analysis of variance model was used to evaluate the differences in the variations of postoperative levels of aspartate aminotransferase, creatinine, and total bilirubin over time.

\section{Results}

\section{Demographic characteristics}

The significant differences between the mild and moderate HCA groups were not identified in term of age, sex, incidence of malperfusion, acute TAAD, cardiac tamponade, Marfan syndrome, and cardiac function. The incidence of chronic TAAD and moderate or severe aortic regurgitation were higher in the moderate HCA group $(21.3 \%$ vs. $4.9 \%$, $\mathrm{P}=0.031 ; 38.3 \%$ vs. $17.1 \%, \mathrm{P}=0.028$, respectively).

\section{Operative data}

The operative data and surgical procedures are listed in Table 2. Compared with the moderate HCA group, the mild HCA group had a significantly higher core temperature (nasopharynx: $24.4 \pm 0.8$ vs. $28.5 \pm 2, \mathrm{P}<0.001$; bladder $25.9 \pm 0.9$ vs. $30 \pm 1.2, \mathrm{P}<0.001)$. The distribution of the intimal tear in TAAD patients was comparable between the two groups. No differences existed between the groups in the incidence of hemiarch replacement, TAR with SET implantation, and concurrent procedures, such as ascending aorta replacement, aortic sinus repair, bypass, the Bentall procedure, and coronary artery bypass grafting (CABG). Unilateral SCP was applied in $44(93.6 \%)$ patients in the moderate HCA group, while bSCP was performed in 35 cases $(85.4 \%)$ in the mild HCA group. Compared to the mild HCA group, patients in the moderate HCA group were characterized by a markedly longer duration for all of the following: CPB time $(221.8 \pm 54.3$ vs. $186.7 \pm 55.3$, $\mathrm{P}=0.004)$, cross-clamp time $(130 \pm 24.9$ vs. $106.8 \pm 33$, $\mathrm{P}<0.001)$, HCA time $(25.8 \pm 10.4$ vs. 19.8 $\pm 9.5, \mathrm{P}=0.006)$, and SCP time (36.8 \pm 8.3 vs. $21.1 \pm 10.7, \mathrm{P}<0.001)$.

\section{Perioperative mortality and morbidity}

Table 3 shows the details of the postoperative outcomes. Compared with the moderate HCA group, the incidence of MAE in the mild HCA group was notably lower [10 (21.3\%) vs. 2 (4.9\%), $\mathrm{P}=0.031]$. No significant differences were identified in the proportions of inhospital mortality between the groups, with 4 deaths $(8.5 \%)$ recorded in the moderate HCA group and 1 death $(2.4 \%)$ in the mild HCA group $(\mathrm{P}=0.366)$. The reasons for mortality in the moderate HCA group were as follows: low cardiac output syndrome in two patients treated by TAR and SET implantation, pulmonary embolism in one patient with hemiarch replacement, and disseminated intravascular coagulation in one patient with TAR and SET implantation. In the mild HCA group, one patient developed severe myocardial infarction 1 day after TAR and SET implantation.

New postoperative neurological complications, such as PND, occurred in four patients in the moderate HCA group, while there were no such occurrences in the mild HCA group $(8.5 \%$ vs. $0, \mathrm{P}=0.12)$. Of the four patients with PND, two experienced right cerebral infarction, and bilateral cerebral infarction developed in one, and left cerebral infarction in one. The incidence of TND in the moderated HCA group was $6.4 \%(3 / 47)$, and $7.3 \%$ (3/41) in the mild HCA group $(\mathrm{P}=1.0)$. Of the six patients that experienced TNDs, all experienced agitation, and temporary delirium occurred in two patients in the mild HCA group. All of these TNDs resolved completely before 
Table 2 Operative outcomes

\begin{tabular}{|c|c|c|c|}
\hline Variables & Moderate HCA group $(n=47)$ & Mild HCA group $(n=41)$ & $P$ value \\
\hline Lowest nasal temperature & $24.4 \pm 0.8$ & $28.5 \pm 2$ & $<0.001$ \\
\hline \multicolumn{4}{|l|}{ Location of intimal tear in TAAD } \\
\hline Ascending aorta & $21 / 46(45.7 \%)$ & $22 / 38(57.9 \%)$ & 0.264 \\
\hline Descending aorta & $6 / 46(13 \%)$ & $1 / 38(2.6 \%)$ & 0.121 \\
\hline Multiple tears & $4 / 46(8.7 \%)$ & 0 & 0.123 \\
\hline Unidentified & $1 / 46(2.2 \%)$ & 2/38 (5.3\%) & 0.587 \\
\hline Hemiarch replacement & $8(17 \%)$ & $2(4.9 \%)$ & 0.097 \\
\hline bSCP & $3(6.4 \%)$ & $35(85.4 \%)$ & $<0.001$ \\
\hline Operation time, $\mathrm{h}$ & $7.1 \pm 1.2$ & $7.1 \pm 1.5$ & 0.916 \\
\hline CPB time, $\min$ & $221.8 \pm 54.3$ & $186.7 \pm 55.3$ & 0.004 \\
\hline Cross-clamp time, min & $130 \pm 24.9$ & $106.8 \pm 33$ & $<0.001$ \\
\hline Lower body circulatory arrest time, min & $25.8 \pm 10.4$ & $19.8 \pm 9.5$ & 0.006 \\
\hline Selective cerebral perfusion time & $36.8 \pm 8.3$ & $21.1 \pm 10.7$ & $<0.001$ \\
\hline Aortic sinus repair & $18(38.3 \%)$ & $8(19.5 \%)$ & 0.054 \\
\hline Ascending aorta replacement & $32(68.1 \%)$ & $28(68.3 \%)$ & 0.983 \\
\hline
\end{tabular}

HCA, hypothermic circulatory arrest; uSCP, unilateral selective cerebral perfusion; bSCP, bilateral selective cerebral perfusion; CPB, cardiopulmonary bypass; CABG, coronary artery bypass grafting.

discharge.

No significant differences were identified in the rate of paraplegia, AKI, tracheotomy, and re-exploration for bleeding. The rate of renal failure requiring CRRT was higher in the moderate HCA group (12.8\% vs. $0, \mathrm{P}=0.028)$. Compared with the moderate HCA group, the duration of ventilator support was shorter in the mild HCA group $\{15[12,19]$ vs. $12[10,14], \mathrm{P}=0.004\}$, as was the duration of the intensive care unit (ICU) stay $\{18.3[14,59.8] \mathrm{vs}$. $16.7[13,26], \mathrm{P}=0.034\}$. The volume of drainage during the first $24 \mathrm{~h}$ was lower in the mild HCA group (748.7 \pm 415.8 vs. $601.7 \pm 223, \mathrm{P}=0.042)$, as was the amount of platelets transfusion $[0(0,1) v s .0(0,0), \mathrm{P}=0.009]$.

\section{Perioperative visceral organ functions}

As shown in Figures 1-3, a significant postoperative escalation in the levels of aspartate aminotransferase, total bilirubin, and creatinine was noticed in both groups $(\mathrm{P}<0.05)$. Aspartate aminotransferase and total bilirubin reached their peak concentrations on postoperative day 2 and day 1, respectively, in both groups. Creatinine reached its peak concentration on postoperative day 1 in the moderate HCA group and on day 2 in the mild HCA group. The perioperative trends in the levels of aspartate aminotransferase, total bilirubin, and creatinine did not differ between the groups, as revealed by mixed effect 
Table 3 Postoperative results

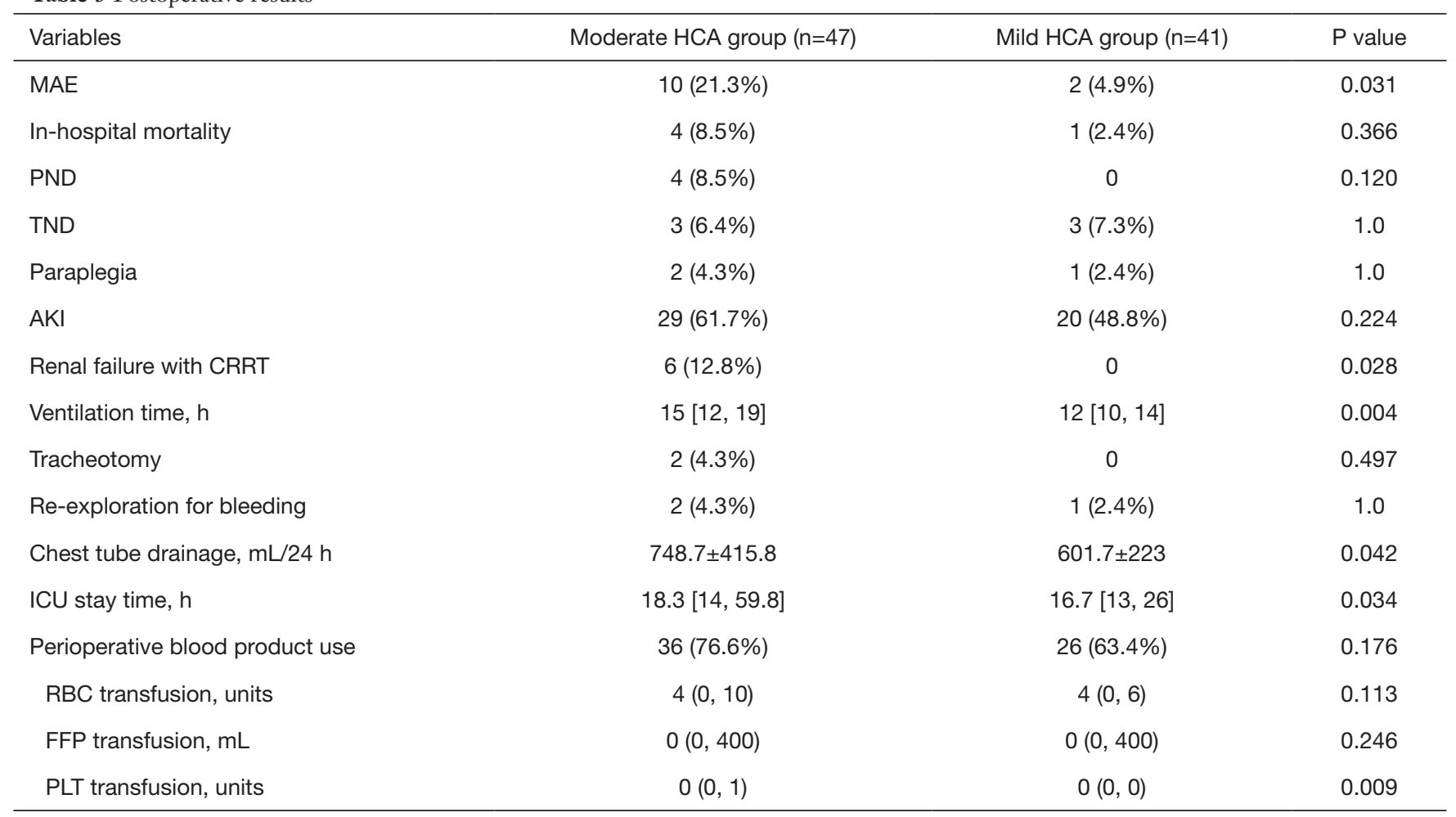

Data presented as mean $\pm \mathrm{SD}$, interquartile ranges, or percentages as appropriate. HCA, hypothermic circulatory arrest; MAE, major adverse events; PND, permanent neurologic deficit; TND, temporary neurological deficit; AKI, acute kidney injury; CRRT, continuous renal replacement therapy; ICU, intensive care unit; RBC, red blood cell; FFP, fresh frozen plasma; PLT, platelets.

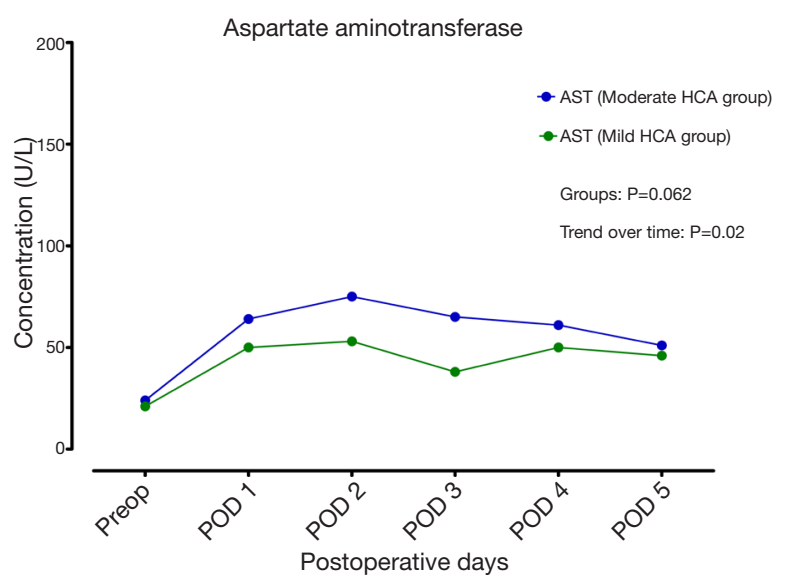

Figure 1 Changes of serum AST concentration with time in the moderate and mild HCA groups. AST, aspartate aminotransferase; HCA, hypothermic circulatory arrest.

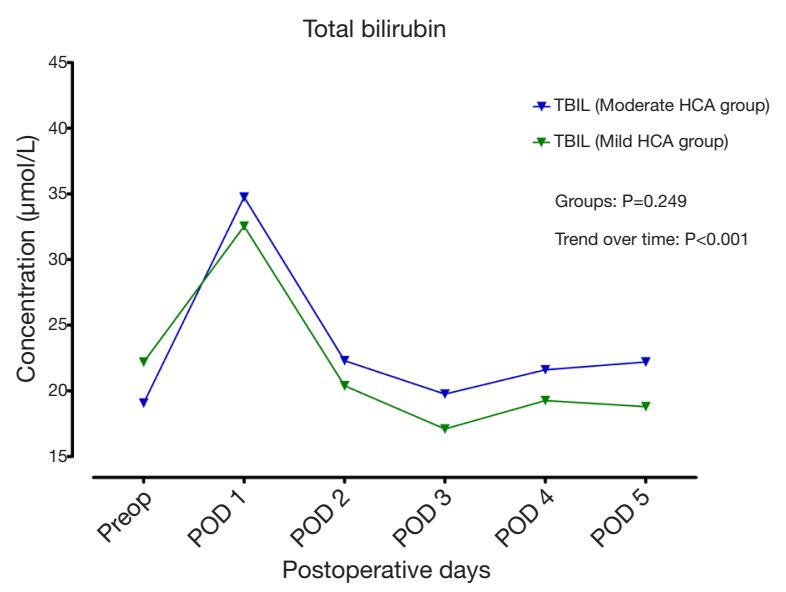

Figure 2 Changes of serum total bilirubin (TBIL) concentration with time in the moderate and mild HCA groups. HCA, hypothermic circulatory arrest. 


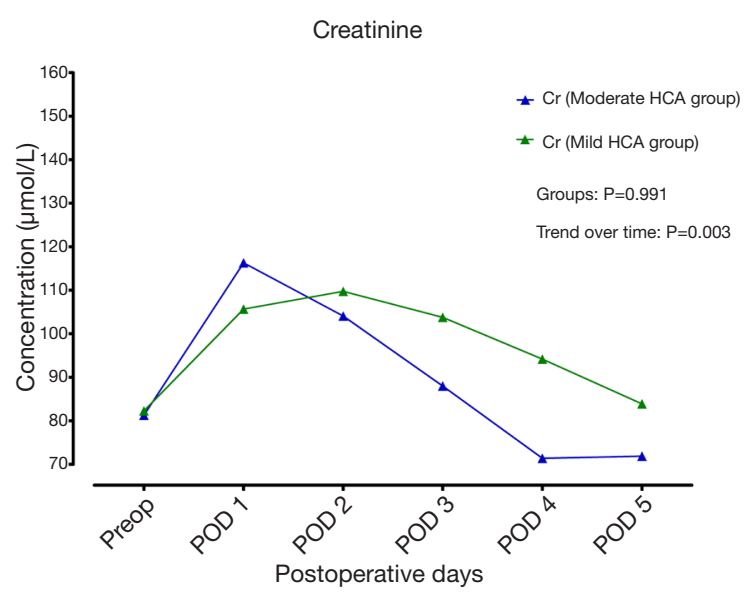

Figure 3 Changes of serum creatinine concentration with time in the moderate and mild HCA groups. HCA, hypothermic circulatory arrest.

analysis of variance modeling (all $\mathrm{P}>0.05$ ).

\section{Follow-up}

By December 2020, follow-up was completed for all survivors, including forty patients in the mild HCA group and 43 cases in the moderate HCA group, with an average of $27 \pm 14$ months (range, 3 to 47 months). During followup, no patients received further intervention in either of the groups, and no major cardiac or cerebral adverse events occurred.

\section{Discussion}

Presently, there is disagreement regarding the optimal temperature of deep HCA in complicated arch surgery. Deep hypothermia limits systemic metabolism and provides a bloodless operative field in open arch repair. However, the benefits of deep HCA could be attenuated by the prolonged $\mathrm{CPB}$ time contributing to cooling and rewarming. Recently, some benefits of deep HCA have been disputed due to prolonged CPB time, limited safe time of distal arch anastomosis, a higher incidence of neurological dysfunction, and clotting disturbances (12). Harel et al. found that more than $180 \mathrm{~min}$ of CPB could not only cause a 3 - to 4-fold increase in the risk of AKI, but could also elevate in-hospital mortality significantly (13). In a comparative study performed by Fang et al. (14), patients with deeper hypothermia showed an increased requirement for platelet transfusion.
To relieve the severe complications of deep HCA and enhance the safe duration of arch procedure, various SCP and HCA strategies have been attempted, and have shown acceptable results $(6,15,16)$. In our institute, we used direct right axillary artery cannulation as our primary $\mathrm{CPB}$ and unilateral cerebral protection strategy in open arch procedures under moderate HCA, with satisfactory results in perioperative mortality and neurological morbidity over a long period of time $(11,17,18)$. To reduce the aforementioned side effects of deep HCA, we attempted mild HCA in open arch repair assisted by uSCP or bSCP, mainly in TAAD patients treated with TAR and SET implantation.

In the mild HCA group, one acute TAAD patient with cardiac tamponade developed lasting ventricular fibrillation after removing the cross-clamp during a TAR and SET implantation procedure. Considering the prominent plaque in the anterior descending branch (revealed by aortic CTA), coronary stenosis was suspected, and a concomitant grafting to the anterior descending branch was performed. Despite subsequent extracorporeal membrane oxygenation being applied after grafting, the patient eventually died of circulatory failure 2 days after surgery.

PND did not develop in the mild HCA group, while four cerebral infarctions (8.5\%) occurred in the moderate HCA group. Three TNDs were noticed in each group. In this study, the perioperative mortality and morbidity from neurological disorders was comparable to that in the literature. We believe that several factors contribute to this. Firstly, this cohort of patients was relatively young. The atherosclerotic changes in the aortic wall were not severe, and the preoperative comorbidities were low. Secondly, according to our institutional policy as an aortic referral center, many acute TAAD patients with poor conditions, such as cardiac tamponade with unstable circulation, myocardial infarction, or severe malperfusion, may die on the way to hospital. Thirdly, the antegrade SCP was a more physiological and effective method of cerebral protection. During proximal root manipulations, the blood flow was retrograde from the cannulation site to the arch, which may contribute to reduced embolic events in the right hemisphere.

Cerebral protection during arch surgery is still a controversial issue. Over the past decade, the acceptance of SCP, in combination with moderate-to-mild systemic hypothermia clearly increased. With respect to the more suitable SCP strategies in open arch procedures, the relative benefits of uSCP compared with bSCP remain 
undetermined. A previous study of the application of unilateral and bilateral SCP in acute TAAD patients concluded that both strategies were equally effective for cerebral protection, and USCP was recommended for its simplicity and less manipulation of arch branch vessels (19). The meta-analysis performed by Malvindi et al. (20), which consisted of 2,949 bSCPs and $599 \mathrm{uSCPs}$, found that bSCP allowed for longer SCP time, with increasing safety once the SCP time exceeded 40-50 min. In a study focusing on TAAD with TAR performed by Tong et al., the bSCP did not demonstrate a significantly lower 30-day mortality or PND rate compared with uSCP (21). In a study including 1,081 patients from the German Registry for aTAAD, they found that the early postoperative neurologic results with bSACP and USACP were similar (22).

In our experience, uSCP was more convenient to establish, and no additional cannula was placed in the operating field. Bilateral SCP represented a more physiologic perfusion strategy, and was related to a reduced risk of hypoperfusion in left hemisphere in patients with an incomplete Willis circle. Also, another manipulation in the left common carotid artery was necessary, and we did not encounter embolic events related to cannulating the left carotid artery.

In this study, patients with mild HCA were characterized by reduced CPB, cross-clamp, and SCP time, which may be explained by the difference in the hypothermia level and sequence of aortic reconstruction to a large extent. Compared to the moderate HCA group, the lowest bladder temperature was markedly higher in patients with mild hypothermia, which contributed to a reduced cooling and rewarming time. For patients that underwent TAR with SET implantation assisted by bSCP, which was the major cerebral perfusion strategy in this group, we resumed the cardiac perfusion upon implantation of a SET and distal arch anastomosis. In contrast to cases with uSCP under moderate hypothermia, we completed the left common carotid artery first to resume bilateral cerebral perfusion after distal arch manipulations, and then performed cardiac perfusion. Thus, we speculate that the higher core temperature and modified sequence of aortic reconstruction helped to shorten the duration of CPB, cross-clamp, and SCP time. The clotting disorders related to prolonged $\mathrm{CPB}$ were also alleviated, which was evidenced by the notably reduced volume of drainage during the first $24 \mathrm{~h}$ and reduced platelet transfusion in the mild HCA group. This finding was similar to the study by Keenan et al., who reported that prolonged $\mathrm{CPB}$ time was related to a higher risk of postoperative bleeding, such that more platelets were required (23).

Compared with cerebral protection by hypothermia and SCP, the distal viscera and spinal cord had a significant risk of ischemia due to single hypothermic protection, especially in the context of constantly increasing core temperature in open arch repair. Acute renal failure was the most frequent marker of visceral organ damage identified in aortic surgery. The incidence of AKI after TAR with SET implantation has been reported to be as high as $77.6 \%$ (24). AKI could be a crucial factor related to increased mortality and morbidity after arch repair (25). In a retrospective study performed by Fang et al., which focused on the differences in the incidence of AKI between different hypothermic strategies (deep or moderate) in a cohort of 627 patients undergoing TAR (14), it was concluded that moderate and deep HCA had a comparable effect on AKI after TAR in TAAD, and that hypothermic difference was not a predictor of AKI. Also, Zhou et al. reported that patients may benefit from moderate HCA rather than deep HCA due to the modifiability of the CPB duration (24). In this study, the incidence of AKI between the moderate and mild HCA groups was not statistically significant $(61.7 \%$ vs. $48.8 \%$, $\mathrm{P}=0.224)$, although a lower incidence of $\mathrm{AKI}$ was observed in the mild HCA group. Moreover, the perioperative trends of the level of creatinine did not differ between the groups, as revealed by mixed effect analysis of variance modeling $(\mathrm{P}=0.991)$.

Motomura et al. reported the rate of acute renal failure requiring dialysis was $7 \%$ in a cohort of 4,707 patients undergoing thoracic aortic surgery (26). In the present study population, renal failure requiring CRRT did not develop in the mild HCA group, while six patients received CRRT in the moderate HCA group (12.8\% vs. $0, \mathrm{P}=0.028$ ). We believe that the incidence of kidney injury in cases with mild hypothermia was comparable to that in the moderate HCA group.

There is still no consensus regarding the safe limit of ischemic tolerance of the spinal cord during HCA at a higher temperature. The Mount Sinai group reported that spinal cord ischemic tolerance was significantly prolonged when cooling to mild hypothermia $\left(32^{\circ} \mathrm{C}\right)$ prior to aortic cross-clamping. Mild hypothermia substantially increased the spinal cord ischemic tolerance to up to $50 \mathrm{~min}$ (27). Zierer et al. reported only one case of paraplegia under mild hypothermia with SCP in a cohort of 245 cases undergoing arch surgery (28). A further study by Zierer et al. involving 1,002 patients who underwent hemiarch or TAR via SCP 
(with a mean core temperature of $30^{\circ} \mathrm{C}$ ) reported that the rates of early mortality and paraplegia were $5 \%$ and $0.3 \%$, respectively (6). They believed that warmer cerebral perfusion might help to improve the collateral flow from the brain to the spinal cord, which was proved by the substantial backflow of blood in the descending aorta necessitating continuous suction during open arch repair. This blood backflow may contribute to the protection of the spinal cord.

In our clinical practice, we also observed that the volume of blood backflow in the descending aorta was considerably higher in patients with mild HCA, and another suction continuously placed in the descending aorta was mandatory. In contrast, intermittent suction was enough to keep a clear operative field during distal arch anastomosis in cases with moderate HCA.

In this study, paraplegia developed in 2 patients $(4.3 \%)$ from the moderate HCA group, and in 1 patient $(2.4 \%)$ from mild HCA group, which was consistent with our previous reports $(17,18,29)$. Delayed paraplegia occurred in one male 3 days after a TAR with SET implantation under mild HCA; this patient recovered well 1-week after cerebrospinal fluid drainage. We speculated that rapid thrombus formation in the false lumen, from which the main intercostal artery originated, may have been the major cause in this case, rather than the hypothermic strategy or distal open repair.

A higher incidence of hepatic injury related to the mild hypothermia strategy was not observed in this study, and was verified by the fact that the trends of level of aspartate aminotransferase and total bilirubin did not differ between the groups (all $\mathrm{P}>0.05$ ). Above all, mild HCA assisted by SCP applied in open arch repair provided a similar inferior visceral and spinal cord protection compared with that of the conventional moderate HCA strategy. We believe that the duration of circulatory arrest was a key factor related to the ischemic injury of lower body end organs. El-Sayed Ahmad et al. reported an acceptable organ protection effect in a cohort of elderly patients (mean age 68 years) with open arch repair by moderate-to-mild systemic hypothermia, and the mean circulatory arrest time was $46 \mathrm{~min}$ (30). In cases where the duration of arch repair exceeded $60 \mathrm{~min}$, they inserted a balloon-tipped cannula into the descending aorta for lower body perfusion.

In this study, the mean HCA time was $25.8 \mathrm{~min}$ in the moderate HCA group, and $19.8 \mathrm{~min}$ in the mild HCA group. A markedly shorter duration of HCA was observed, which may be explained by the special surgical strategy. The classic Sun's procedure was applied for most patients in this study, and the lower body was re-perfused upon completion of distal arch anastomosis. In most cases, the anastomosis between the four-branched prosthetic graft and the distal arch containing the intraluminal stented graft required less than $30 \mathrm{~min}$. The pathological distal arch was sandwiched by the unstented part of the SET and the four-branched graft. After a continuous suture, additional reinforced sutures were not usually needed.

The duration of ventilator support and ICU stay was significantly lower in the mild HCA group compared to the moderate HCA group, which may be related to the difference in hypothermia level. Obviously, more evidence is needed to confirm this in the future.

\section{Limitations}

This study has some limitations that should be noted. Due to the retrospective nature of the study, we identified associations as opposed to causalities for all of the evaluated relationships. Secondly, this was a single-center study with a relatively small number of patients. Thirdly, the average age of patients was considerably younger compared to Western cohorts. Finally, long-term outcomes were lacking, and therefore, further investigations are required.

\section{Conclusions}

In this study, mild HCA with SCP was safely applied for open arch repair in a cohort with various arch pathologies. The preliminary results for mild HCA were acceptable, and a lower incidence of MAEs was observed compared to patients that received moderate HCA. Similar inferior outcomes were also obtained with mild HCA compared to the conventional moderate HCA strategy. These encouraging surgical and postoperative results support this more aggressive hypothermia strategy in open arch repair.

\section{Acknowledgments}

Funding: None.

\section{Footnote}

Reporting Checklist: The authors have completed the STROBE reporting checklist. Available at http://dx.doi. org/10.21037/jtd-20-3550

Data Sharing Statement: Available at http://dx.doi. 
org/10.21037/jtd-20-3550

Conflicts of Interest: All authors have completed the ICMJE uniform disclosure form (available at http://dx.doi. org/10.21037/jtd-20-3550). The authors have no conflicts of interest to declare.

Etbical Statement: The authors are accountable for all aspects of the work in ensuring that questions related to the accuracy or integrity of any part of the work are appropriately investigated and resolved. All procedures performed in this study involving human participants were in accordance with the Declaration of Helsinki (as revised in 2013). This study was approved by the Ethics Committee of Beijing Anzhen Hospital, Capital Medical University. Written informed consent was obtained from each patient.

Open Access Statement: This is an Open Access article distributed in accordance with the Creative Commons Attribution-NonCommercial-NoDerivs 4.0 International License (CC BY-NC-ND 4.0), which permits the noncommercial replication and distribution of the article with the strict proviso that no changes or edits are made and the original work is properly cited (including links to both the formal publication through the relevant DOI and the license). See: https://creativecommons.org/licenses/by-nc-nd/4.0/.

\section{References}

1. Griepp RB, Stinson EB, Hollingsworth JF, et al. Prosthetic replacement of the aortic arch. J Thorac Cardiovasc Surg 1975;70:1051-63.

2. Kazui T, Washiyama N, Muhammad BA, et al. Total arch replacement using aortic arch branched grafts with the aid of antegrade selective cerebral perfusion. Ann Thorac Surg 2000;70:3-8; discussion 8-9.

3. Bachet J, Guilmet D, Goudot B, et al. Antegrade cerebral perfusion with cold blood: a 13-year experience. Ann Thorac Surg 1999;67:1874-8; discussion 1891-4.

4. Bachet J, Guilmet D, Goudot B, et al. Cold cerebroplegia. A new technique of cerebral protection during operations on the transverse aortic arch. J Thorac Cardiovasc Surg 1991;102: 85-93; discussion 93-4.

5. Tian DH, Wan B, Bannon PG, et al. A meta-analysis of deep hypothermic circulatory arrest versus moderate hypothermic circulatory arrest with selective antegrade cerebral perfusion. Ann Cardiothorac Surg 2013;2:148-58.

6. Zierer A, El-Sayed Ahmad A, Papadopoulos N, et al.
Selective antegrade cerebral perfusion and mild $\left(28^{\circ} \mathrm{C}\right.$ $30^{\circ} \mathrm{C}$ ) systemic hypothermic circulatory arrest for aortic arch replacement: results from 1002 patients. J Thorac Cardiovasc Surg 2012;144:1042-9.

7. Tokuda Y, Miyata H, Motomura N, et al. Brain protection during ascending aortic repair for Stanford type A acute aortic dissection surgery. Nationwide analysis in Japan. Circ J 2014;78:2431-8.

8. Yan TD, Bannon PG, Bavaria J, et al. Consensus on hypothermia in aortic arch surgery. Ann Cardiothorac Surg 2013;2:163-8.

9. Kellum JA, Lameire N. Diagnosis, evaluation, and management of acute kidney injury: a KDIGO summary (Part 1). Crit Care 2013;17:204.

10. Liu ZG, Sun LZ, Chang Q, et al. Should the "elephant trunk" be skeletonized? Total arch replacement combined with stented elephant trunk implantation for Stanford type A aortic dissection. J Thorac Cardiovasc Surg 2006;131:107-13.

11. Sun L, Qi R, Zhu J, et al. Total arch replacement combined with stented elephant trunk implantation: a new "standard" therapy for type a dissection involving repair of the aortic arch? Circulation 2011;123:971-8.

12. Di Eusanio M, Wesselink RMJ, Morshuis WJ, et al. Deep hypothermic circulatory arrest and antegrade selective cerebral perfusion during ascending aorta-hemiarch replacement: a retrospective comparative study. J Thorac Cardiovasc Surg 2003;125:849-54.

13. Harel Z, Bell CM, Dixon SN, et al. Predictors of progression to chronic dialysis in survivors of severe acute kidney injury: a competing risk study. BMC Nephrol 2014; 15:114.

14. Fang Z, Wang G, Liu Q, et al. Moderate and deep hypothermic circulatory arrest has a comparable effect on acute kidney injury after total arch replacement with frozen elephant trunk procedure in type A aortic dissection. Interact Cardiovasc Thorac Surg 2019;29:130-6.

15. Estrera AL, Miller CR, Lee TY, et al. Ascending and transverse aortic arch repair: the impact of retrograde cerebral perfusion. Circulation 2008;118:S160-6.

16. Zierer A, Aybek T, Risteski P, et al. Moderate hypothermia $\left(30^{\circ} \mathrm{C}\right)$ for surgery of acute type A aortic dissection. Thorac Cardiovasc Surg 2005;53:74-9.

17. Sun LZ, Qi RD, Chang Q, et al. Surgery for acute type A dissection using total arch replacement combined with stented elephant trunk implantation: experience with 107 patients. J Thorac Cardiovasc Surg 2009;138:1358-62.

18. Chen Y, Ma WG, Li JR, et al. Can Frozen Elephant Trunk 
Cure Type I Dissection Confined to Thoracic Aorta in Marfan Syndrome? Ann Thorac Surg 2020;109:1174-82.

19. Norton EL, Wu X, Kim KM, et al. Unilateral is comparable to bilateral antegrade cerebral perfusion in acute type A aortic dissection repair. J Thorac Cardiovasc Surg 2020;160:617-625.e5.

20. Malvindi PG, Scrascia G, Vitale N. Is unilateral antegrade cerebral perfusion equivalent to bilateral cerebral perfusion for patients undergoing aortic arch surgery? Interact Cardiovasc Thorac Surg 2008;7:891-7.

21. Tong G, Zhang B, Zhou X, et al. Bilateral versus unilateral antegrade cerebral perfusion in total arch replacement for type A aortic dissection. J Thorac Cardiovasc Surg 2017;154:767-75.

22. Krüger T, Weigang E, Hoffmann I, et al. Cerebral protection during surgery for acute aortic dissection type a: results of the German registry for acute aortic dissection type a (GERAADA). Circulation 2011;124:434-43.

23. Keenan JE, Wang H, Gulack BC, et al. Does moderate hypothermia really carry less bleeding risk than deep hypothermia for circulatory arrest? A propensity-matched comparison in hemiarch replacement. J Thorac Cardiovasc Surg 2016;152:1559-1569.e2.

24. Zhou H, Wang G, Yang L, et al. Acute Kidney Injury After Total Arch Replacement Combined With Frozen Elephant Trunk Implantation: Incidence, Risk Factors, and Outcome. J Cardiothorac Vasc Anesth 2018;32:2210-17.

25. Ko T, Higashitani M, Sato A, et al. Impact of Acute

Cite this article as: Dong SB, Zhang K, Zhu K, Wang LF, Zheng J, Li JR, Liu YM, Sun LZ, Pan XD. Mild hypothermic circulatory arrest with selective cerebral perfusion in open arch surgery. J Thorac Dis 2021;13(2):1151-1161. doi: 10.21037/jtd-203550
Kidney Injury on Early to Long-Term Outcomes in Patients Who Underwent Surgery for Type A Acute Aortic Dissection. Am J Cardiol 2015;116:463-8.

26. Motomura N, Miyata H, Tsukihara H, et al. Risk model of thoracic aortic surgery in 4707 cases from a nationwide single-race population through a web-based data entry system: the first report of 30-day and 30-day operative outcome risk models for thoracic aortic surgery. Circulation 2008;118:S153-9.

27. Strauch JT, Lauten A, Spielvogel D, et al. Mild hypothermia protects the spinal cord from ischemic injury in a chronic porcine model. Eur J Cardiothorac Surg 2004;25:708-15.

28. Zierer A, Detho F, Dzemali O, et al. Antegrade cerebral perfusion with mild hypothermia for aortic arch replacement: single-center experience in 245 consecutive patients. Ann Thorac Surg 2011;91:1868-73.

29. Ma WG, Zheng J, Zhang W, et al. Frozen elephant trunk with total arch replacement for type A aortic dissections: Does acuity affect operative mortality? J Thorac Cardiovasc Surg 2014;148:963-70; discussion 970-2.

30. El-Sayed Ahmad A, Papadopoulos N, Risteski P, et al. The Standardized Concept of Moderate-to-Mild $\left(\geq 28^{\circ} \mathrm{C}\right)$ Systemic Hypothermia During Selective Antegrade Cerebral Perfusion for All-Comers in Aortic Arch Surgery: Single-Center Experience in 587 Consecutive Patients Over a 15-Year Period. Ann Thorac Surg 2017;104:49-55. 